\title{
Assessment of the Visual Analogue Score in the Evaluation of the Pruritus of Cholestasis
}

\author{
Nora V. Bergasa*1,2 and E. Anthony Jones ${ }^{\# 3}$ \\ ${ }^{1} N Y C, H+H$ Metropolitan, New York, NY, USA; ${ }^{2}$ New York Medical College, Valhalla, NY, USA; ${ }^{3}$ Department of \\ Gastroenterology and Liver Diseases, Academic Medical Center, Amsterdam, The Netherlands
}

\begin{abstract}
Background and Aims: A visual analogue score (VAS), based on application of a visual analogue scale, has been widely used to assess pruritus in clinical studies of patients with cholestatic liver disease. A VAS is a numerical score of the severity of the perception of pruritus, and, hence, is inherently subjective. The objective of this study was to assess the reliability of a VAS as an index of pruritus in cholestatic patients. Methods: In 8 patients with chronic pruritus due to primary biliary cholangitis, values for a VAS of pruritus were compared with corresponding measurements of scratching activity, which were generated by a monitoring system specifically designed to quantitate this activity. The relationship between individual values for the VAS and corresponding values for scratching activity during a specific interval immediately preceding the recording of the VAS was examined by determining the Spearman's rank correlation coefficient. Results: The mean Spearman's rank correlation coefficient between individual values for the VAS and corresponding mean values for scratching activity was 0.072 ; the range of these coefficients was -0.04 to 0.26 . A VAS of pruritus is an unreliable index of scratching activity, and, hence, of the pathophysiological process responsible for the pruritus of cholestasis. Conclusion: It is concluded that the use of a VAS as a primary quantitative endpoint in trials of the efficacy of potential therapies for the pruritus of cholestasis may be inappropriate.
\end{abstract}

Citation of this article: Bergasa NV, Jones EA. Assessment of the visual analogue score in the evaluation of the pruritus of cholestasis. J Clin Transl Hepatol 2017;5(3):203-207. doi: 10.14218/JCTH.2017.00001.

\section{Introduction}

The clinical syndrome of cholestasis is a complication of many hepatobiliary diseases. The syndrome arises as a consequence of impaired hepatocellular secretion of bile from any cause. It is characterized by an accumulation in plasma of substances that are normally secreted into bile, such as

Keywords: Pruritus; Cholestasis; Visual analogue score; Scratching activity; Primary biliary cholangitis; Quantitative efficacy endpoint.

Abbreviations: VAS, visual analogue score.

Received: 7 January 2017; Revised: 20 May 2017; Accepted: 23 May 2017 \#Deceased.

*Correspondence to: Nora V. Bergasa, NYC, H + H, Metropolitan 1901 First Avenue, New York, NY 10128, USA. Tel: +1-212-423-6771, Fax: +1-212-4238099, E-mail: bergasan2@nychhc.org bilirubin, bile acids and cholesterol. ${ }^{1,2}$ A distressing symptom in many patients with cholestasis is pruritus. This symptom may be severe and intractable, and associated with sleep deprivation and suicidal ideations. Relief from this symptom is often difficult to achieve. Indeed, intractable pruritus of cholestasis may be an indication for liver transplantation. ${ }^{3}$ A notable feature of this form of pruritus, in contrast to many others, is that it is not readily relieved by scratching. The pathogenesis of the pruritus of cholestasis is unknown. ${ }^{4-6}$

In accordance with the basic principles of clinical science, it has long been recognized that progress in studying factors that relieve or exacerbate this form of pruritus would be facilitated by applying an appropriate objective quantitative method to its assessment. Such an approach would be likely to provide insights into the pathogenesis of the pruritus and, when used as an endpoint in clinical trials, would provide a means for assessing the efficacy of potential therapies. In this context, a visual analogue score (VAS) of pruritus, based on the application of a visual analogue scale (Fig. 1), appears to have been widely accepted as a method of assessing pruritus in clinical studies of chronic cholestasis. ${ }^{7-13}$ However, the reliability and objectivity of the numerical data generated from the inherently subjective marks made by cholestatic patients on visual analogue scales do not seem to have been critically evaluated.

As scratching is a consequence of pruritus, an alternative method of assessing the severity of the pruritus of cholestasis, which is independent of any subjective input from patients, is to measure directly the intensity of scratching activity. In this study, the relationship between measurements of the perception of pruritus, as assessed from a VAS, and scratching activity, as assessed by applying a monitoring system specifically designed to measure this activity (Fig. 2), ${ }^{14}$ was evaluated in patients with pruritus due to the chronic cholestatic disease, primary biliary cirrhosis. The results have implications for the optimal design of clinical trials of potential therapies for pruritus.

\section{Methods}

\section{Patients}

Eight female patients with chronic pruritus secondary to the chronic cholestatic disease, primary biliary cholangitis, formerly known as primary biliary cirrhosis, were studied. The patients had been participants of a pilot controlled trial of naloxone infusions for the pruritus of cholestasis. ${ }^{15}$ The design was a single-blind, placebo controlled study of naloxone infusions administered in a non-predetermined order. ${ }^{15}$ 
No itch

Worst itch ever

Fig. 1. A visual analogue scale consisting of a $\mathbf{1 0 -} \mathrm{cm}$ horizontal line. The origin of the line on the left side is designated "no itch" and the end of the line on the right side is designated "worst itching ever". To indicate the severity of itch, a patient is requested to make a mark on the scale that corresponds to the relative severity of itch currently being experienced. This mark is made with a pen or pencil. The visual analogue score (VAS) is the number of centimeters to the nearest millimeter between the origin of the scale on the left side and the mark on the scale made by the patient. ${ }^{15}$

In none of the patients had the pruritus been relieved satisfactorily by scratching or by conventional medications that are commonly prescribed for this type of pruritus, such as orally administered resins (e.g., cholestyramine, colestipol), antihistamines or phenobarbital. ${ }^{1,6}$ Their ages ranged from 46 to 62 years.

For each patient, results of routine serum biochemical tests were indicative of cholestasis over a period of at least 2 years, and a needle biopsy of the liver revealed histological features characteristic of primary biliary cholangitis. ${ }^{16}$ The serum of all but one of the patients was positive for antibodies to mitochondria. Each patient gave informed consent to participate in the study. All antipruritic medications were discontinued at least 5 days before the onset of the study. Before entering the study, each patient was observed to scratch with their fingernails at frequent intervals.

\section{Descriptions of pruritus}

The patients' descriptions of their perception of pruritus varied substantially. For example, the sensation was described as "biting" by one patient, "pins and needles" by another, and as a burning and intolerable feeling sometimes like "taking the skin off" by another. In most patients the pruritus was generalized, but in one it was localized to the chest, face and scalp. One patient was self-conscious about her itching and was concerned that other people might think that her scratching indicated that her condition was contagious; she stated that her itching was worse when she went home from work and that it was less of a problem when she was working and distracted by specific activities. In one patient the pruritus tended to be more severe at the time of menstruation; she had an unsatisfactory marital relationship and her perception of severe pruritus was sometimes rapidly and dramatically relieved by a change of environment (e.g., emergency admission to hospital). Another patient also had an unsatisfactory marital situation and she was skeptical that she would derive any benefit from medical therapy for her pruritus, feeling that only liver transplantation would alleviate the symptom. These descriptions highlight the subjectivity of the perception of the pruritus of cholestasis and the heterogeneous nature of its impact on different patients.

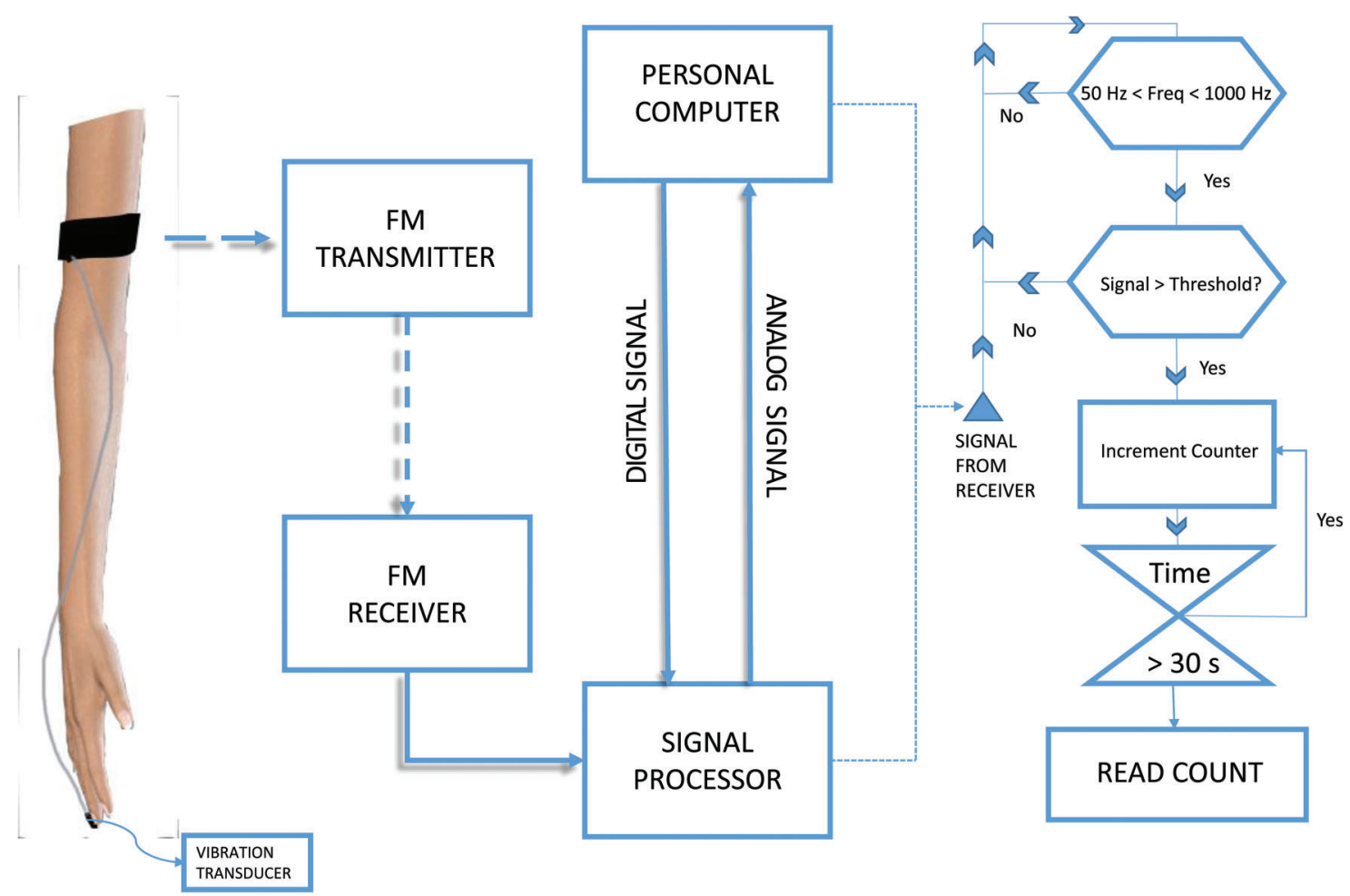

Fig. 2. Block diagram of the scratching activity monitoring system. The system consists of a vibration (scratch) transducer, an FM transmitter and receiver, a custombuilt signal processor and a personal computer. The vibration transducer is taped to the middle fingernail of the dominant hand. The transducer consists of a square piece of piezoelectric film, 28- $\mu \mathrm{m}$ thick, metalized on both sides with silver ink. It is connected by wire to a transmitter that is attached to the adjacent arm by a Velcro cuff. The transducer converts the strain produced by vibrations of the fingernail as it traverses the skin in the act of scratching into an electrical voltage. The electrical signal is transmitted across the room where it is received, processed and logged by a computer. Frequencies associated with vibrations of the scratching fingernail were found to be between 30 and $1000 \mathrm{~Hz}$, whereas those associated with gross body movements were $<30 \mathrm{~Hz}$. The device is programmed to exclude signals with frequencies of <30 $\mathrm{Hz}$, so that scratching activity independent of arm or hand movements was recorded as a scratching activity index, with units of counts per unit time. ${ }^{13}$ The average scratching activity for consecutive 15-minute intervals was recorded. 
Bergasa N.V. et al: Visual analogue scale and scratching behavior

\section{VASs}

Every 4 waking-hours, patients were asked to record the average severity of their pruritus during the preceding 4-hour period by making a mark with a pen or pencil on a visual analogue scale. The scale consisted of a $10-\mathrm{cm}$ horizontal line, with $0-\mathrm{cm}$ point (beginning of scale on the left side) being designated as representing no itching and the $10-\mathrm{cm}$ point (end of scale on the right side) representing worst ever itching (Fig. 1). ${ }^{17} \mathrm{~A}$ VAS is the number of centimeters, to the nearest millimeter, between $0 \mathrm{~cm}$ and the point on the scale where the patient made a mark.

\section{Index of scratching activity}

An objective numerical index of scratching activity was generated by applying a monitoring system specifically designed to measure this activity. ${ }^{14}$ Scratching activity was continuously recorded for the duration of the study, which was conducted in the in-patient research unit. Briefly, the system consisted of a vibration (scratch) transducer, an FM transmitter and receiver, a custom-built signal processor and a personal computer (Fig. 2). The vibration transducer was taped to the middle fingernail of the dominant hand; this fingernail was not cut during the study. The transducer consisted of a square piece of piezoelectric film, $28-\mu \mathrm{m}$ thick, metalized on both sides with silver ink. It was connected by wire to a transmitter that was attached to the adjacent arm by a Velcro cuff. The transducer converted the strain produced by vibrations of the fingernail as it traversed the skin in the act of scratching into an electrical voltage.

The electrical signal was transmitted across the room, where it was received, processed and logged by a computer. Frequencies associated with vibrations of the scratching fingernail were found to be between 30 and $1000 \mathrm{~Hz}$, whereas those associated with gross body movements were $<30 \mathrm{~Hz}$. The device was programmed to exclude signals with frequencies of $<30 \mathrm{~Hz}$, so that scratching activity independent of arm or hand movements was recorded as a scratching activity index, with units of counts per unit time. ${ }^{14}$ The average scratching activity for consecutive 15-minute intervals was recorded. For this study, scratching activity was recorded continuously for 2 to 4 successive 24 -hour periods.

\section{Statistical analysis}

Recordings of scratching activity during 4-hour periods immediately prior to individual determinations of a VAS were analyzed. The first VAS after waking was not included in the analysis because of the possible effects of sleep on the perception of itch. The relationship between individual values for the VAS and corresponding values for scratching activity during a specific interval immediately preceding the recording of the VAS was examined by determining the Spearman's rank correlation coefficient $\left(r_{\mathrm{s}}\right)$. Specifically, the set of VAS values recorded by each patient was correlated with the corresponding set of values for scratching activity recorded during the immediately preceding 15-minute period. Similar correlations were also determined between VAS values and mean values for scratching activity recorded during the immediately preceding 30-, 60-, 120- and 240-minute intervals. Four to 11 sets of these comparisons were made for each patient.

\section{Results}

Spearman's rank correlation coefficients between individual values for the VAS and mean values for scratching activity recorded during five different time intervals immediately preceding the recording of the VAS, varied between 0.04 and 0.26 (Table 1 ). The overall mean correlation coefficient was 0.072 . Thus, in this study population with primary biliary cholangitis, the severity of the perception of pruritus, as assessed by a VAS, did not correlate with an objective quantitative index of scratching activity.

\section{Discussion}

Pruritus, or itching, is a major complication of cholestasis. Itch is defined as the need to scratch and, hence, is intrinsically subjective. As itch is a perception, it cannot be measured directly. Itch has long been assessed subjectively by the use of questionnaires and descriptive terms, such as mild, moderate and severe. It has also been assessed by determination of a VAS, which enables the perception of itch to be represented by a numerical value, and, hence, gives the impression that the severity of pruritus has been quantitated. Whether such numbers represent an adequate or meaningful index of pruritus has not been rigorously evaluated. Nevertheless,

Table 1. Spearman's rank correlation coefficients between individual values for the visual analogue score (VAS) and mean values for scratching activity recorded over 15, 30, 60, 120 and 240 minutes prior to the time point at which the visual analogue scale, from which the VAS was calculated, was marked

\begin{tabular}{lllllll}
\hline Patient number & Number of VASs per patient & $0-15^{\prime}$ & $0-30^{\prime}$ & $0-60^{\prime}$ & $0-120^{\prime}$ & $0-240^{\prime}$ \\
\hline 1 & 4 & -0.80 & -0.80 & -0.80 & -0.80 & -0.20 \\
2 & 11 & -0.20 & -0.18 & 0.14 & -0.01 & -0.01 \\
3 & 4 & 0.80 & 0.40 & 0.40 & 0.20 & 1.00 \\
4 & 8 & -0.01 & 0.07 & -0.10 & 0.16 & 0.02 \\
5 & 6 & -0.44 & -0.14 & 0.54 & 0.37 & 0.43 \\
6 & 9 & 0.10 & 0.13 & 0.08 & 0.44 & 0.24 \\
7 & 10 & 0.19 & 0.10 & -0.12 & 0.01 & 0.12 \\
8 & 10 & 0.14 & 0.69 & 0.46 & 0.63 & 0.46 \\
Average correlation coefficient & & -0.04 & 0.03 & 0.08 & 0.13 & 0.26 \\
\hline
\end{tabular}


determination of a VAS appears to be a well-established research method for assessing pruritus in patients with cholestasis. ${ }^{7-13}$ VAS has also been extensively applied in psychology and other medical disciplines, in which it has been used to assess other specific symptoms, such as pain. ${ }^{17-19}$ However, an important issue is whether a VAS constitutes a sufficiently reliable index of the severity of pruritus for its application to be useful in clinical studies of cholestatic patients.

In this context, it is probably worth carefully reconsidering potential problems with interpreting a VAS that were highlighted in a landmark critical review by McCormack et al. ${ }^{17}$ Issues of concern include the following: (i) There tends to be a tri-modal distribution of data points with clusters at the midpoint and extremes of the visual analogue scale; (ii) Interpatient variability in marking the scales is evident; (iii) An ability to transform a complex subjective experience into a visual-spatial display, which involves perceptual judgement and accuracy, is required; (iv) A variety of factors contribute to respondent error, such as age, ability to think abstractly, mental organization, and perceptual skills; ( $v$ ) It is necessary to examine how scales were marked on previous occasions; and ( $\mathrm{vi}$ ) Early use of the maximal value precludes subsequent accurate scoring of a greater perception. ${ }^{17}$

A VAS is, therefore, affected by inter-individual variations in the ability of patients to integrate the perception of itch over time and to translate the outcome of this process into a visual-spatial score. The conceptualization of such a score is also subject to inter-patient differences. ${ }^{17}$ In addition, marking a visual analogue scale is subject to changes due to hand and hand preferences, ${ }^{20}$ and the act of making a mark on a horizontal line, such as a visual analogue scale, can be influenced by sex. ${ }^{21}$ Thus, a variety of factors may potentially contribute to uncertainty concerning the validity of assessments of perceptions obtained by marking a visual analogue scale. ${ }^{17}$ Nevertheless, VAS has been utilized extensively as an endpoint in clinical trials designed to determine the efficacy of potential therapies for the pruritus of cholestasis. ${ }^{7-13}$ The discipline of clinical science requires that an endpoint in a therapeutic trial be, not only relevant, but also quantitative and objective. The question arises whether a VAS meets these requirements in trials of potential therapies for the pruritus of cholestasis.

To address this issue, it is necessary to compare values of a VAS with objective measurements of another variable that directly reflects the pathophysiology of the pruritus of cholestasis. As scratching can be defined as the behavioral consequence of pruritus, quantitative data on scratching activity, which are independent of any subjective input from patients, may fulfil this requirement. The first serious attempt to measure scratching activity in pruritic patients was reported over three decades ago, when a device was developed for measuring limb movements in patients with pruritic skin disorders. ${ }^{22}$ Subsequently, a more sophisticated apparatus was designed to measure limb movements of patients with pruritus due to chronic cholestasis. ${ }^{23}$ The development of such an apparatus reflected the need for objective quantitative data in clinical studies of this syndrome. The major deficiency of these original methods was that measurements of limb movements record activities other than scratching activity. This problem was subsequently overcome by developing the well-tolerated device used in this study, which measures scratching activity independent of hand or limb movements. ${ }^{14}$
The validity of the scratching activity monitoring system used in this study has been verified by demonstrating a close concordance between its output (numerical values of a scratching activity index or the intensity of an audio-signal proportional to these values) and independent direct observations and video recordings of scratching activity by the fingernail bearing the transducer. In particular, counts were not recorded by the device during gross movements of the relevant hand that were not associated with an interaction between fingernails and the skin or during scratching activity by the hand that did not bear the transducer. ${ }^{14}$ This monitoring system enables the behavioral consequence of the pruritus of cholestasis to be detected and measured irrespective of whether patients complain of pruritus or perceive its sensation. Thus, in contrast to the sensation of itch, scratching activity can be directly and objectively measured. Furthermore, the relevance of measuring scratching is supported by finding a significant 24-hour rhythm of this activity in patients with the pruritus of cholestasis, ${ }^{24}$ a finding which suggests that scratching activity in such patients is under central control and is a direct consequence of the pathophysiological process responsible for the pruritus of cholestasis.

In contrast to this study, previous studies of patients with the pruritus of cholestasis, in which both assessments of a VAS and measurements of scratching activity were made, ${ }^{15,24-26}$ were not designed primarily to study the relationship between these two variables but to evaluate the efficacy of either intravenously administered naloxone ${ }^{15,24}$ or orally administered nalmefene ${ }^{25,26}$ in ameliorating pruritus. Accordingly, the relationship between VAS data and measurements of scratching activity was not a primary endpoint in these previous studies. It follows that that their design did not enable the relationship between VAS data and measurements of scratching activity to be rigorously evaluated; and, hence, the need for a more definitive study in which this relationship was itself the primary endpoint was not precluded. The current study was designed to fulfil this objective and, consequently, its findings are not directly comparable to those in any of the previous studies. ${ }^{15,24-26}$

Although it has long been claimed that itching correlates strongly with scratching activity, ${ }^{25}$ this study indicates that the intensity of the perception of itch, as assessed by a VAS, does not correlate with an objective quantitative index of scratching activity in patients with pruritus due to primary biliary cholangitis. Thus, a VAS, as applied in this study did not provide a reliable assessment of the magnitude of the pathophysiological process that is responsible for inducing scratching activity in our study population (women). Accordingly, the results of this study do not provide support for the use of a VAS as an endpoint in clinical trials of potential therapies for the pruritus of cholestasis.

Notwithstanding its manifest limitations, VAS has been popular as an endpoint in this context, ${ }^{7-13}$ presumably because it is so easy to apply in clinical studies and investigators may not have been familiar with, or prepared to develop, any more appropriate alternative. It would appear that the measurement of scratching activity is such an alternative, and that a strong case can be made for including measurements of scratching activity as an endpoint in future clinical trials designed to determine the efficacy of potential therapies for the pruritus of cholestasis. Failure to include measurements of scratching activity in the design of such studies on the grounds of the complexity, impracticality or expense of a scratching activity monitoring system cannot be justified. 
In the case of the monitor used in this study, ${ }^{14}$ its simplicity would readily be confirmed by any bioengineer shown its block diagram (Fig. 2), it is easy to apply and well-tolerated by patients, and its components are either cheap, such as piezofilm, or readily available, such as personal computers.

\section{Acknowledgments}

The author is most grateful to the late Dr. David W. Alling for his authoritative advice on statistical analysis of the data. The National Institutes of Health, Intramural Funding, Liver Unit provided funding for this project. Ethical approval was obtained, as cited in Reference 15.

\section{Conflict of interest}

The authors have no conflict of interests related to this publication.

\section{Author contributions}

Designed the study, collected and interpreted the data and wrote the paper, and contributed equally to this manuscript (NVB, EAJ).

\section{References}

[1] Sherlock S. Overview of chronic cholestatic conditions in adults: terminology and definitions. Clin Liver Dis 1998;2:217-233, vii. doi: 10.1016/S10893261(05)70004-0.

[2] Reichen J, Simon FR. Mechanisms of cholestasis. Int Rev Exp Pathol 1984; 26:231-274.

[3] Neuberger J, Jones EA. Liver transplantation for intractable pruritus is contraindicated before an adequate trial of opiate antagonist therapy. Eur J Gastroenterol Hepatol 2001;13:1393-1394. doi: 10.1097/00042737-20011100000022.

[4] Jones EA, Bergasa NV. The pruritus of cholestasis. Hepatology 1999;29: 1003-1006. doi: 10.1002/hep.510290450.

[5] Bergasa NV. Pruritus in primary biliary cirrhosis: pathogenesis and therapy. Clin Liver Dis 2008;12:385-406; x. doi: 10.1016/j.cld.2008.02.013.

[6] Bergasa NV. Pruritus of cholestasis. In: Carstens E, Akiyama T, editors. Itch: Mechanisms and treatment. Frontiers in neuroscience. Boca Raton (FL); 2014.

[7] Ghent CN, Carruthers SG. Treatment of pruritus in primary biliary cirrhosis with rifampin. Results of a double-blind, crossover, randomized trial. Gastroenterology 1988;94:488-493. doi: 10.1016/0016-5085(88)90442-8.

[8] Woolf GM, Reynolds TB. Failure of rifampin to relieve pruritus in chronic liver disease. J Clin Gastroenterol 1990;12:174-177. doi: 10.1097/00004836199004000-00012.

[9] Wolfhagen FH, Sternieri E, Hop WC, Vitale G, Bertolotti M, Van Buuren HR. Oral naltrexone treatment for cholestatic pruritus: a double-blind, placebocontrolled study. Gastroenterology 1997;113:1264-1269. doi: 10.1053/ gast.1997.v113.pm9322521.
[10] Müller C, Pongratz S, Pidlich J, Penner E, Kaider A, Schemper M, et al. Treatment of pruritus in chronic liver disease with the 5-hydroxytryptamine receptor type 3 antagonist ondansetron: a randomized, placebo-controlled, double-blind cross-over trial. Eur J Gastroenterol Hepatol 1998;10: 865-870. doi: 10.1097/00042737-199810000-00010.

[11] O'Donohue JW, Pereira SP, Ashdown AC, Haigh CG, Wilkinson JR, Williams R. A controlled trial of ondansetron in the pruritus of cholestasis. Aliment Pharmacol Ther 2005;21:1041-1045. doi: 10.1111/j.1365-2036.2005.02430.x.

[12] Rifai K, Hafer C, Rosenau J, Athmann C, Haller H, Peter Manns M, et al. Treatment of severe refractory pruritus with fractionated plasma separation and adsorption (Prometheus). Scand J Gastroenterol 2006;41:1212-1217. doi: $10.1080 / 00365520600610154$

[13] Parés A, Herrera M, Avilés ], Sanz M, Mas A. Treatment of resistant pruritus from cholestasis with albumin dialysis: combined analysis of patients from three centers. J Hepatol 2010;53:307-312. doi: 10.1016/j.jhep. 2010.02.031.

[14] Talbot TL, Schmitt JM, Bergasa NV, Jones EA, Walker EC. Application of piezo film technology for the quantitative assessment of pruritus. Biomed Instrum Technol 1991;25:400-403.

[15] Bergasa NV, Talbot TL, Alling DW, Schmitt JM, Walker EC, Baker BL, et al. A controlled trial of naloxone infusions for the pruritus of chronic cholestasis. Gastroenterology 1992;102:544-549. doi: 10.1016/00165085(92)90102-5.

[16] Scheuer PJ. Ludwig Symposium on biliary disorders-part II. Pathologic features and evolution of primary biliary cirrhosis and primary sclerosing cholangitis. Mayo Clin Proc 1998;73:179-183. doi: 10.1016/S0025-6196 (11)63652-5.

[17] McCormack HM, Horne DJ, Sheather S. Clinical applications of visual analogue scales: a critical review. Psychol Med 1988;18:1007-1019. doi: 10 . $1017 /$ S0033291700009934.

[18] Kostic MA, Gutierrez FJ, Rieg TS, Moore TS, Gendron RT, A prospective, randomized trial of intravenous prochlorperazine versus subcutaneous sumatriptan in acute migraine therapy in the emergency department. Ann Emerg Med 2010;56:1-6. doi: 10.1016/j.annemergmed.2009.11.020.

[19] Gladwin MT, Kato GJ, Weiner D, Onyekwere OC, Dampier C, Hsu L, et al. Nitric oxide for inhalation in the acute treatment of sickle cell pain crisis: a randomized controlled trial. JAMA 2011;305:893-902. doi: 10 . 1001/jama.2011.235.

[20] McKechnie JG, Brodie EE. Hand and hand preferences in use of a visual analogue scale. Percept Mot Skills 2008;107:643-650. doi: 10.2466/pms. 107.3.643-650.

[21] Hausmann $M$, Ergun G, Yazgan $Y$, Güntürkün $O$. Sex differences in line bisection as a function of hand. Neuropsychologia 2002;40:235-240. doi: 10.1016/S0028-3932(01)00112-9.

[22] Felix R, Shuster S. A new method for the measurement of itch and the response to treatment. $\mathrm{Br}$ J Dermatol 1975;93:303-312. doi: 10.1111/j. 1365-2133.1975.tb06496.x.

[23] Summerfield JA, Welch ME. The measurement of itch with sensitive limb movement meters. Br J Dermatol 1980;103:275-281. doi: 10.1111/j. 1365-2133.1980.tb07244.x.

[24] Bergasa NV, Alling DW, Talbot TL, Swain MG, Yurdaydin C, Turner ML, et al. Effects of naloxone infusions in patients with the pruritus of cholestasis. A double-blind, randomized, controlled trial. Ann Intern Med 1995;123: 161-167. doi: 10.7326/0003-4819-123-3-199508010-00001.

[25] Bergasa NV, Alling DW, Talbot TL, Wells MC, Jones EA. Oral nalmefene therapy reduces scratching activity due to the pruritus of cholestasis: a controlled study. J Am Acad Dermatol 1999;41:431-434. doi: 10.1016/S01909622(99)70117-9.

[26] Bergasa NV, Schmitt JM, Talbot TL, Alling DW, Swain MG, Turner ML, et al. Open-label trial of oral nalmefene therapy for the pruritus of cholestasis. Hepatology 1998;27:679-684. doi: 10.1002/hep.510270307. 